\title{
Observations of Exciton Density of State Variations in a ZnO Thin Film with fs Pump-probe Experiments
}

\author{
Cheng-Yen Chen, Yen-Cheng Lu, Hsiang-Chen Wang, Fang-Yi Jen, and C. C. Yang \\ Graduate Institute of Electro-Optical Engineering and Department of Electrical Engineering. \\ National Taiwan University, 1, Roosevelt Road, Sec. 4, Taipei, Taiwan, R.O.C. \\ cricucenenticilutw \\ Bao-ping Zhang and Yusaburo Segawa \\ Photodaynamics Research Center. The Institute of Physical and Chemical Research (RIKEN). Sendai, Japan
}

\begin{abstract}
Variations of exciton density of state around the levels of donor-bound and free excitons in a $\mathrm{ZnO}$ thin film are observed with temperature- and photon energy-dependent degenerate fs pump-probe spectroscopy for exploring its exciton dynamics.

2005 Optical Society of America

OCIS codes: (320.7150) Ultrafast spectroscopy; (160.6000) Semiconductors, including MQW
\end{abstract}

\section{Introduction}

The radiative recombination of an electron and a hole in an exciton is more efficient than those of free motion. Therefore, for developing efficient light-emitting devices, compound materials of larger exciton binding energies are more attractive. Because of the large exciton binding energy ( $60 \mathrm{meV}$ versus $30 \mathrm{meV}$ in $\mathrm{GaN})$ in $\mathrm{ZnO}$, it has attracted quite much attention in crystal growth and optical characterization, including ultrafast carrier dynamics [1-4]. In such a compound, excitons exist in several forms, including donor-bound exciton $\left(D^{0} \mathrm{X}\right)$ and free exciton (FX). Since the high photon emission efficiency of such a compound relies on exciton dynamics, the understandings of exciton density of state distribution and hence ultrafast exciton dynamics are crucially important. In this paper, we observe the variation of exciton density of state and explore the exciton dynamics in a $\mathrm{ZnO}$ thin film with fs pump-probe spectroscopy. In particular, we perform temperature- and photon energy-dependent measurements around the $\mathrm{D}^{0} \mathrm{X}$ and FX levels. Exciton flow among these levels can be evaluated.

\section{Sample Preparation and Experimental Methods}

The sample was grown in an MOCVD reactor on sapphire substrate. The growth temperature was $450{ }^{\circ} \mathrm{C}$ for 60 min [5]. The growth pressure was 6 Torr. The in-plane orientation could be controlled with the growth temperature, which could lead to different growth modes at the initial stage of growth. With the aforementioned growth condition, $30^{\circ}$ in-plane twist was obtained in the used sample. The second-harmonic generation through a BBO crystal of a fs Ti:sapphire laser with $100 \mathrm{fs}$ in pulse width and $76 \mathrm{MHz}$ in pulse repetition frequency was used for the degenerate pump-probe experiments. The second-harmonic pulse width was about $150 \mathrm{fsec}$. The pump power was maintained at about $16 \mathrm{~mW}$ and the probe power was one tenth of the pump power.

\section{Pump-probe Experimental Results}

Temperature dependent photoluminescence (PL) spectra of the sample are shown in Fig. 1. Here, one can see that the $\mathrm{PL}$ peak of increasing width red shifts with temperature. At $10 \mathrm{~K}$, only one major peak of $\mathrm{D}^{0} \mathrm{X}$ (at $3.365 \mathrm{eV}$ ) can be seen. However, at $20 \mathrm{~K}$ a small hump around $3.376 \mathrm{eV}$, corresponding to the emergence of FX, can be observed. This hump becomes more prominent and dominant in spectra beyond $80 \mathrm{~K}$. It red shifts, particularly significantly beyond $80 \mathrm{~K}$, to $3.293 \mathrm{eV}$ at $300 \mathrm{~K}$ [6]. Beyond $80 \mathrm{~K}$, two peaks at 3.307 and $3.230 \mathrm{eV}$ (at $80 \mathrm{~K}$ ), corresponding to the DAP (donor-acceptor pair) and DAP-LO (LO phonon-assisted DAP), respectively, can be identified. It is noted that the $\mathrm{D}^{0} \mathrm{X}$ feature position does not vary significantly with temperature until it disappears around $100 \mathrm{~K}$. In this temperature range, the $\mathrm{FX}$ feature position is almost fixed. The spectral peak energies of $\mathrm{D}^{0} \mathrm{X}$ and $\mathrm{FX}$ at $10 \mathrm{~K}$ are used for the pump-probe experiments.

Fig. 2 shows the differential transmission profiles at various temperatures when the pump-probe photon energy is $3.366 \mathrm{eV}$, corresponding to the $\mathrm{D}^{0} \mathrm{X}$ state at $10 \mathrm{~K}$. Here, one can see that $\Delta \mathrm{T} / \mathrm{T}$ is significantly strong at 10 and 90 $\mathrm{K}$. Usually the variation magnitude of $\Delta \mathrm{T} / \mathrm{T}$ represents the band filling effect. Hence, the large magnitudes in differential transmission at 10 and $90 \mathrm{~K}$ can be interpreted as high densities of state at the pump-probe photon energies at these two temperatures. As mentioned, this photon energy corresponds to $\mathrm{D}^{0} \mathrm{X}$ state at $10 \mathrm{~K}$. It approximately coincides with the state of $F X$ at $90 \mathrm{~K}$, as shown in Fig. 1. The relatively higher densities of states at the $\mathrm{D}^{0} \mathrm{X}$ and $\mathrm{FX}$ lead to the stronger pump-probe responses. The two-stage decay time constants of these pump-probe profiles are labeled in the figure. The fast decay in the early stage, ranging from 0.8 to $4.3 \mathrm{ps,} \mathrm{describes}$ exciton thermalization through scattering. The relatively slower decay, in the time range of a few tens ps, beyond $100 \mathrm{~K}$ 


\section{CMII5}

explains the mixed process of exciton recombination and mild relaxation into the FX state. The slightly increasing trends in the later stage when temperature is lower than $100 \mathrm{~K}$ (without labeling the decay time constants) may be due to the in-flow of excitons into the corresponding states of either $\mathrm{D}^{0} \mathrm{X}$ or FX level.

Fig. 3 shows the differential transmission profiles at various temperatures when the pump-probe photon energy is $3.376 \mathrm{eV}$, corresponding to the $\mathrm{FX}$ state at $10 \mathrm{~K}$. Basically, the magnitude of $\Delta \mathrm{T} / \mathrm{T}$ decreases with temperature, particularly significantly beyond $100 \mathrm{~K}$. The decreasing trend is due to the increasing gap between the fixed pump-probe photon energy $(3.376 \mathrm{eV})$ and the decreasing FX level as temperature increases. As shown in Fig. 1, this gap becomes particularly significant beyond $100 \mathrm{~K}$. Hence, the differential transmission level decreases faster beyond $100 \mathrm{~K}$. The decay time constants of the early-stage fast decay (in the range of a couple ps) again refer to the process of exciton/carrier thermalization. The time constants labeled for the slower second decay stage mainly describe the relaxation of excitons/carriers into the $\mathrm{FX}$ and $\mathrm{D}^{0} \mathrm{X}$ states.

Fig. 4 shows the $\Delta \mathrm{T} / \mathrm{T}$ profiles of several pump-probe photon energies at $10 \mathrm{~K}$. The first curve from the top $(3.370 \mathrm{eV})$ corresponds to the photon energy between the states of $\mathrm{D}^{0} \mathrm{X}$ and FX. The rest are between the FX level and the conduction band edge. The bottom curve for $3.419 \mathrm{eV}$ may be quite close to the donor level (bound electron), which is estimated to be at $3.425 \mathrm{eV}$. Again, the fast decay here is related to exciton/carrier thermalization. The slower decay involves exciton relaxation into the $\mathrm{D}^{0} \mathrm{X}$ level. In this process, for the pump-probe photon energies close to either the FX or donor level, part of carriers may flow back into the probe level. This mechanism explains the slightly increasing trends in the top curve and the two bottom curves. The oscillation in the curve of $3.419 \mathrm{eV}$ can be due to a coupling process between two levels. It deserves further investigation.

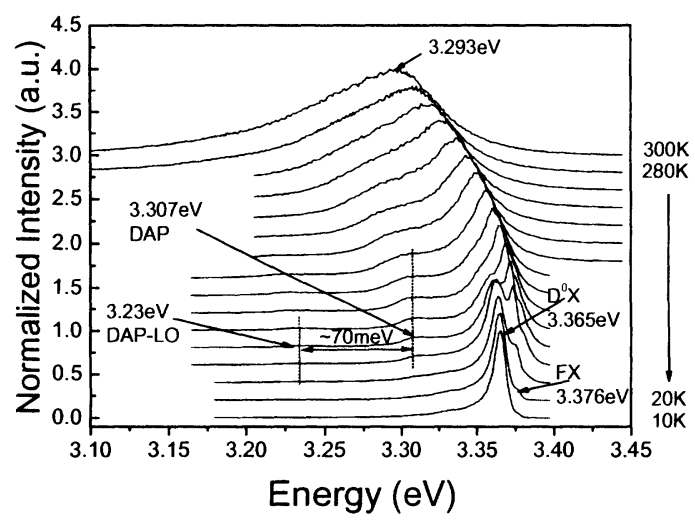

Fig. 1 Temperature-dependent photoluminescence spectra of the sample.

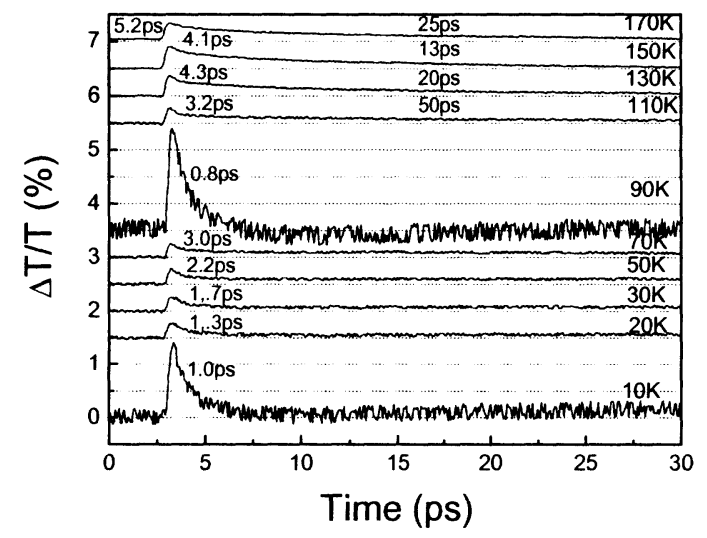

Fig. 2 Differential transmission profiles at various temperatures when the pump-probe photon energy is $3.366 \mathrm{eV}$, corresponding to the $\mathrm{D}^{0} \mathrm{X}$ state at $10 \mathrm{~K}$. 


\section{CMII5}

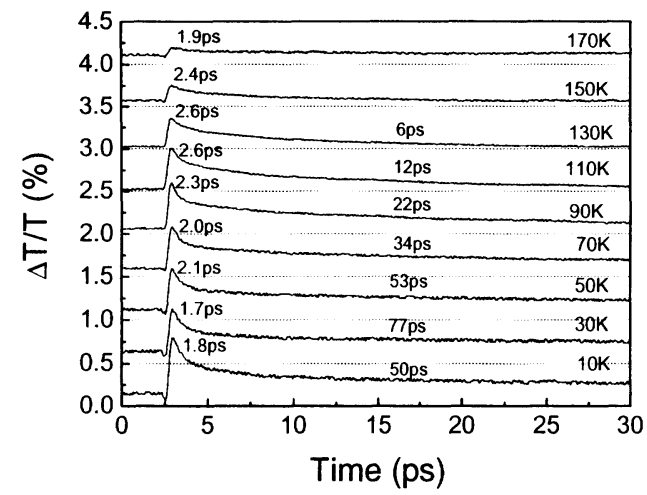

Fig. 3 Differential transmission profiles at various temperatures when the pump-probe photon energy is $3.376 \mathrm{eV}$, corresponding to the FX state at $10 \mathrm{~K}$.

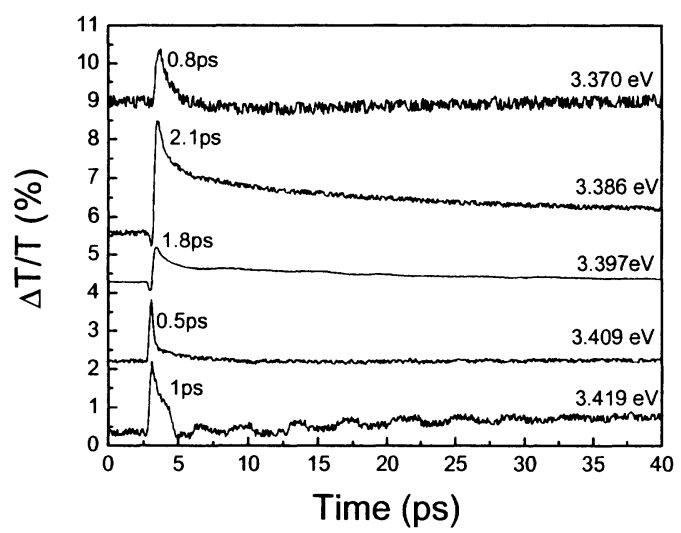

Fig. 4 Differential transmission profiles at several photon energies when the temperature is $10 \mathrm{~K}$.

\section{Conclusions}

In summary, we have investigated the variations of exciton density of state in a $\mathrm{ZnO}$ thin film around the $\mathrm{D}^{0} \mathrm{X}$ and FX levels with temperature- and photon energy-dependent fs pump-probe measurements. The processes of exciton thermalization and relaxation among the levels of $\mathrm{FX}, \mathrm{D}^{0} \mathrm{X}$, and donor state were observed.

\section{Acknowledgement:}

This research was supported by National Science Council, The Republic of China, under the grant of NSC 92-2210-M-002-006 and NSC 92-2215-E-002-010, and by US Air Force under the contract AOARD-04-4026.

\section{References:}

1. A. Zeuner, H. Alves, D. M. Hofmann, B. K. Meyer, M. Heuken, J. Blasing, and A. Krost, Appl. Phys. Lett. 80, 2078-2080 (2002).

2. A. Tsukazaki, A. Ohtomo, M. Kawasaki, T. Makino, C. H. Chia, Y. Segawa, and H. Koinuma, Appl. Phys. Lett. 84, 3858-3860 (2004)

3. A. Yamamoto, T. Kido, T. Goto, Y. Chen, T. Yao, and A. Kasuya, Appl. Phys. Lett. 75, 469-471 (1999).

4. T. Tokizaki, H. Sakai, A. Nakamura, Y. Manabe, S. Hayashi, and T. Mitsuyu, Semicond. Sci. Technol. 10, 1253-1256 (1995)

5. B. P. Zhang, K. Wakatsuki, N.T. Binh, N. Usami, Y. Segawa, Thin Solid Films 449, 12-19 (2004).

6. S. F. Chichibu, A. Tsukazaki, M. Kawasaki, K. Tamura, Y, Segawa, T. Sota, and H. Koinuma, Appl. Phys. Lett. 80, 5860-5862 (2002). 